\title{
Improvement Multidisciplinary Collaborate Optimization based on Simu- lated Annealing and Artificial Neural Networks
}

\author{
Ning Qiang ${ }^{1, *}$ and Yang Zhao ${ }^{2}$ \\ ${ }^{1}$ School of Marine Science and Technology, Northwestern Polytechnical University, Xi' an, 710072, China; ${ }^{2}$ Department \\ of Electronic and Information Technology, Jiangmen Polytechnic, Jiangmen, 529090, China
}

\begin{abstract}
Multidisciplinary Design Optimization (MDO) is the most active fields in the current complex engineering system design. Forcing to the defects of traditional Collaborative Optimization, such as unable to convergence or falling into local optimum, we propose a Collaborate Optimization based on Simulated Annealing and Artificial Neural Networks, (SA-ANN-CO). The SA-ANN-CO algorithm inherit the parallel distribution strategy of tradition Collaborative Optimization, and then establish accuracy Artificial Neural Networks models by Latin Hypercube Experimental design replace the realistic model of sub-disciplines to reduce computing costs and smooth numerical noise. The possibility of falling into local solutions is reduced by using the Simulated Annealing algorithm in system-level. Two classic test examples results show that, SA-ANN-CO algorithm has good robustness and can quickly and effectively converge to the global optimum solution, which provides a effective way for complex engineering systems design.
\end{abstract}

Keywords: Artificial neural networks, multidisciplinary collaborate optimization, simulated annealing.

\section{INTRODUCTION}

A complex engineering system, with multiple professional fields often involving many design variables and constraints, meanwhile, influence or mutual coupling exists among the various disciplines. However, owning to ignoring the relevance of disciplines, traditional serial approaches always falling into the local optimal solution. Eighties of last century, Multidisciplinary Design Optimization (MDO) rise in the aerospace field. Scientists as Sobieski and Kroo have done some pioneering work in this area [1-3]. MDO is a design methodology that utilizes collaborative mechanism to fully explore the interaction between engineering systems design to design the complex products and subsystems. With the development of MDO, A lot of fruitful multidisciplinary algorithm appears. Such as Multidisciplinary Feasible Method (MDF), Concurrent Subspace Optimization (CSSO), Collaborative Optimization (CO), Bi-Level Integrated System Synthesis (BLISS). CO algorithm, proposed by Kroo et al. in 1994 [2] with good autonomy and parallel processing capabilities, has been considered the most promising multidisciplinary optimization algorithms.

Due to the unique form of consistency restraint in system-level, traditional Collaborative Optimization algorithm may incur the problems that the excessive constraints may exist and the number of iterations will increase significantly in the system-level. More severe case is that the optimization results cannot be convergence. To solve these defects, the literature [4] proposed the optimal sensitivity method, using a first-order approximation method increases the likelihood of convergence, but it results in large computational cost and poor robustness. The literature [5] using dynamic penalty function method to translate the system-level optimization problem into unconstrained optimization problems, which improve the efficiency of the system-level optimization problem, but how to select a appropriate penalty factor to ensure the stability of the calculation lead a new problem. In literature [6] genetic algorithm is applied to system-level optimization to overcome the poor reliability of Collaborative Optimization algorithm, but a significant increase in the number of functions calculation cannot be ignored. In literature [7] the penalty function method is introduced discipline level, and the average value of the subsystem variables instead of the system-level variables is used to implement the system optimization, However, this method cannot solve the problem with unequal number of shared variables in discipline-level. The literature [8] proposed to join mix variable in the collaborative optimization framework to solve complex system optimization design problems, this method, although is a novel idea, leading to greater system-level optimization burden. Based on the above analysis, we propose a Collaborate Optimization based on Simulated Annealing and Artificial Neural Networks, (SA-ANN-CO). The accuracy Artificial Neural Networks models are established by Latin Hypercube Experimental design. Two classic test examples are presented to demonstrate the effective of our method.

\section{IMPROVEMENT COLLABORATE OPTIMIZA- TION}

\subsection{Artificial Neural Network Response Surface Tech-} nology

Artificial Neural Networks (ANN) is a complex parallel network structure consisting of a large number of simple 
processing units that connected to each other. Although each unit can only perform simple calculations, the entire network can constitute a highly complex nonlinear system. Fitting curve close to the real situations can be obtained, if using artificial neural networks for data analysis and processing [9]. Kolmogrov's multilayer network mapping existence theorem shows that if the number of input variables of a function is $n$, the number of output variables is $m$, then a three feedback BP neural network can be created with $\mathrm{n}$ neurons in input layer, $2 n+1$ neurons in hidden layer, $m$ neurons in output layer. Meanwhile, any continuous function can be fitted by three feedback BP neural network, as long as nonlinear increasing functions are applied in the hidden layer and linear functions are applied in the input and output layer. The topology structure of BP neural network is shown in Fig. (1). In Fig. (1), $w_{i j}, w_{j k}$ are the weight values to be estimated, $d_{1}, d_{2} \cdots d_{m}$ are the desired output. Optimal weights of the neural networks is obtained through the samples studying way, which use BP algorithm to find out the weights when the error between actual output and the desired output is minimum, Finally, by training the neural network to adjust the unknown factor we can approximate the original function globally. S-type functions are used in the above hidden layer. The response surface fitting function is:

$$
\bar{f}(x)=\sum_{k=1}^{m} w_{j k} \frac{1}{1+e^{-\sum_{i=1}^{n} x_{i} w_{i j}}}
$$

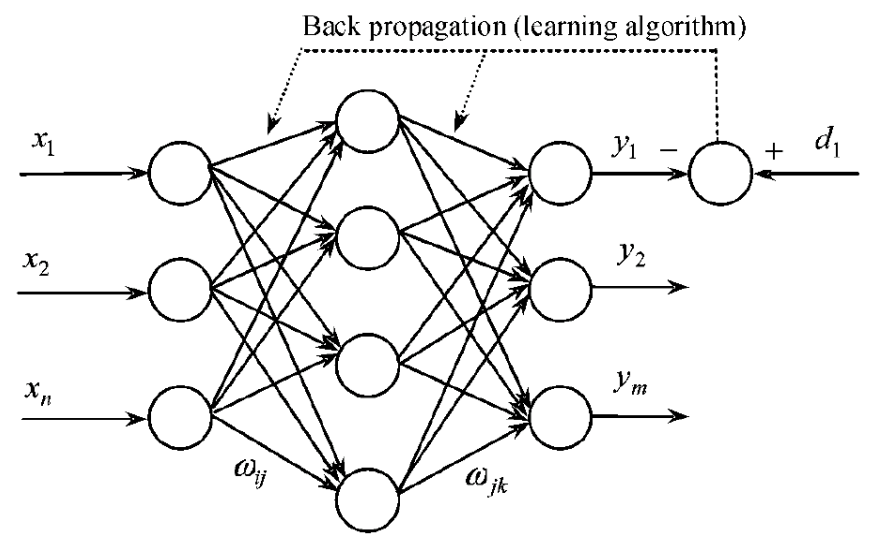

Fig. (1). Three-layer neural network topology diagram.

\subsection{Simulated Annealing Algorithm}

Simulated Annealing derived from the solid simulated principles, core idea of which is similar with the principles of thermodynamics, especially similar to the flow and crystallization of liquid or cooling and annealing of metal. A large number of molecules of the liquid relatively move freely between each other at high temperatures. The thermal energy of the movable will disappears if the liquid cool slowly and then lots of atoms themselves can often be arranged in rows to form a pure crystals, which are orderly arranged within a few hundred times the distance of the individual atoms in the respective directions. Thus, the essence of this process is cool slowly to obtain sufficient time to allow a large number of atoms redistribution before losing of mobility, which is the conditions to ensure low energy state. In simple terms, physical annealing process consists of the following three components $[10,11]$ :

\subsubsection{Heating Process}

Its purpose is to enhance the thermal motion of the particles to deviate them from the equilibrium position. When the temperature is high enough, the solid was melted to a liquid, thus eliminating the system which might exist in a nonuniform state to make the cooling process subsequent to it as a starting point. Entropy of the system link with fusion processes and will rise with temperature increasing.

\subsubsection{Isothermal Process}

Knowledge of physics tells us that when a closed system, with constant temperature, exchange heat with the surrounding environment, the system state is always towards the direction of reducing the free energy and the system reaches equilibrium the minimum free energy.

\subsubsection{Cooling Process}

The aim of cooling process is to weaken the thermal motion of particles and become orderly, with the energy gradual decline in the system, crystal structure with low-energy will be obtained.

The basic steps of SA algorithm are as follows:

1) Generates a random initial solution $x_{0}$, set $x_{\text {best }}=x_{0}$ and calculates the objective function value $E\left(x_{0}\right)$;

2) Set the initial temperature $T(0)=T_{0}$ and the number of the iterations $i=1$;

3) Do while $T(i)>T_{\min }$;

a. for $j=1 \sim k$;

b. for the current optimal solution $x_{\text {best }}$ according to a neighborhood function to generate a new solution $x_{\text {new }}$. Calculate the new objective function value $E\left(x_{\text {new }}\right)$ the incremental value of the objective function $\Delta E=E\left(x_{\text {new }}\right)-E\left(x_{\text {best }}\right)$;

c. if $\Delta E<0$, then $x_{\text {best }}=x_{\text {new }}$;

d. If $\Delta E>0$, then $p=e^{-\Delta E / T(\mathrm{i})}, \quad$ if $c=\operatorname{random}[0,1]<p$, then $x_{\text {best }}=x_{\text {new }}$, else $x_{\text {best }}=x_{\text {best }}$;

e. End for.

4) $i=i+1$;

5) End Do;

6) Output the current optimum and make the algorithm end.

\subsection{SA-ANN-CO and the Solving Process}

The SA-ANN-CO algorithm inherits the parallel distribution strategy of tradition Collaborative Optimization and resolve deficiencies of traditional Collaborative Optimization mainly from three aspects: 
1) As a modern intelligent algorithm, Simulated Annealing algorithm need not special requirements for mathematical expressions of optimization problems and derivative information, even the continuity conditions of design space is no need. Simulated Annealing algorithm is applied in the system level optimization process, which avoiding the problems such as being sensitive to the initial values, falling into local optima and no-convergence.

2) In engineering optimization problems, consistency equality constraints in system are too strictly, we will introduce dynamic slack variables to translate equality constraints into inequality constraints. At the beginning of the optimization, the grant larger slack variable is selected, generally set $10^{-3} \leq \varepsilon_{1} \leq 10^{-1}$. During the optimization process, $\varepsilon=\frac{\varepsilon_{1}}{10 N}$ is necessary, where $\mathrm{N}$ is the number of systemlevel iterations. No doubt, this strategy can accelerate the convergence rate of SA in the application of SA.

3) There are computational complexity, organizational complexity, model complexity and complex of the exchange of information when solve complex systems engineering problem with multi-disciplinary optimization algorithms. Using Artificial Neural Network Response Surface model based on experimental design instead of the true models can effectively reduce optimization cost and reduce the possibility of falling into local optimization solution. At the same time, the approximate model with high precision can smooth design space and guarantee a reasonable solution.

The solving process of SA-ANN-CO is shown in Fig. (2), the details of solving process is as follows:

1) Conducting disciplinary analysis, namely building highly accurate ANN approximation models for the various disciplines. Fig. (3) shows a building flow chart of ANN approximate models. The details of the steps are as follows:

a) Collecting the samples;

b) Generating the ANN response surface models;

c) Verifying the accuracy of the ANN response surface model. To verify the accuracy of the ANN response surface model, we can choose average error, maximum error, residual sum of squares, etc. as an indicator. If the credibility of ANN is enough, the ANN model can be used to replace the real model; otherwise, increasing the sample points.

2) Replacing the real model with ANN response surface models and establish Collaborative Optimization framework;

3) Passing the shared variables in the system-level to the various discipline's meta-models;

4) Based on the ANN response surface models, disciplines should enable the smallest difference between the coupling variables and target values delivered from the system-level under the conditions to meet their constraints;

5) With the consistency constraints, the system level will optimize shared variables to resolve inconsistencies between the disciplines coupled variables and system-level variables to maximize the target.
6) Judging the convergence conditions, if the convergence conditions satisfied, make end to the algorithms, otherwise, returning to the third step.

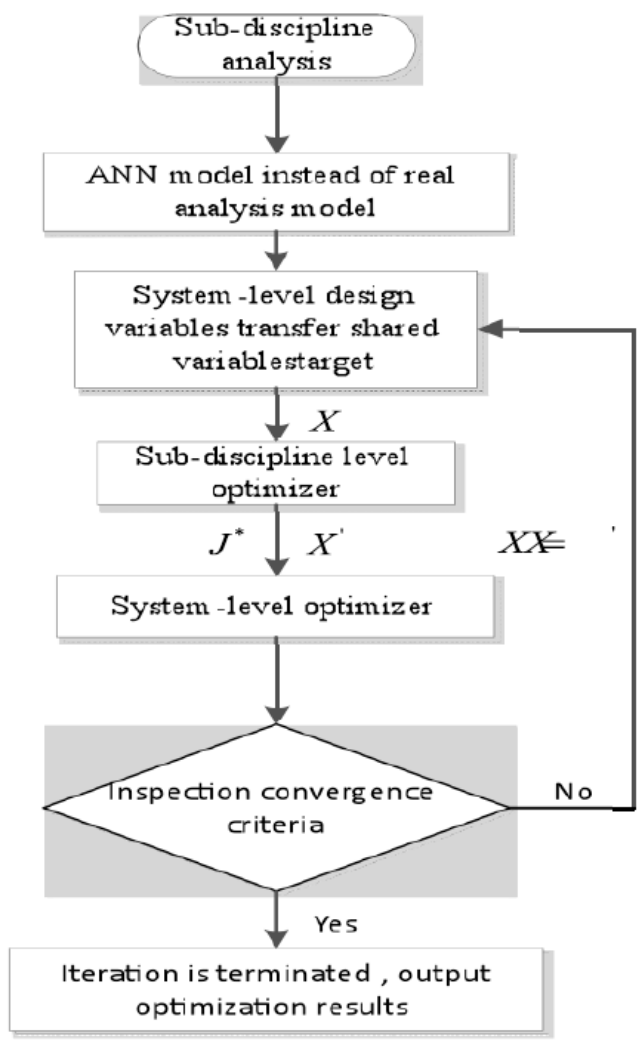

Fig. (2). The solving process of SA-ANN-CO.

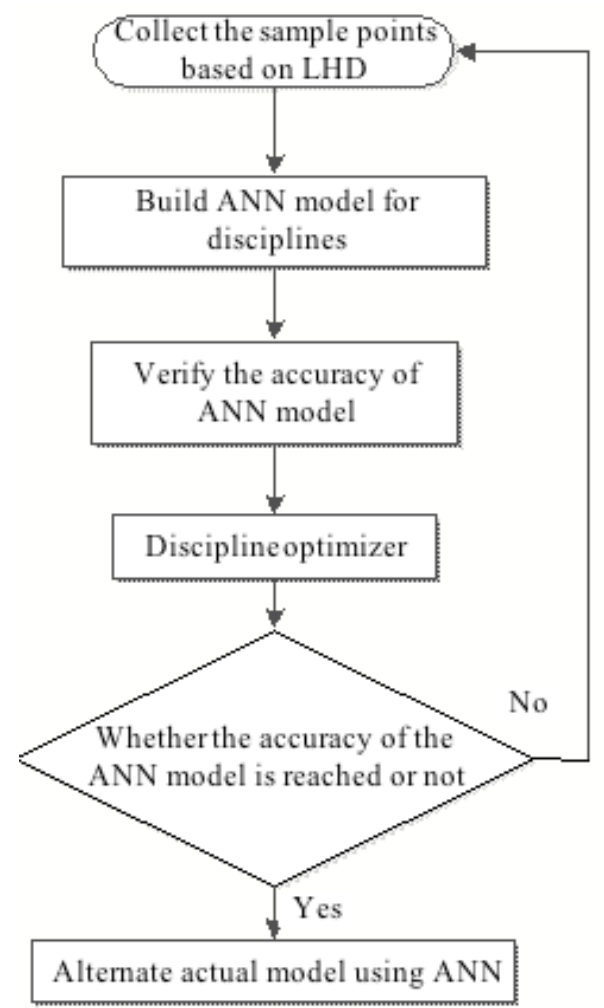

Fig. (3). The building flowchart of ANN. 


\section{EXAMPLES}

In this section we will use two classic MDO test to illustrate the validity and applicability of SA-ANN-CO algorithm.

\subsection{Examples 1}

Example1 is a typical convex optimization problem. The mathematical model is as follows:

$$
\begin{array}{ll} 
& \text { Min : } f(x)=x_{1}^{2}+x_{2}^{2} \\
\text { s.t. } & x_{1}+\beta x_{2}<4 \\
& \beta x_{1}+x_{2}>2
\end{array}
$$

According to the idea of Collaborative Optimization, the optimization problem is divided into a system-level and two disciplines. The mathematical model of Collaborative Optimization is as follows:

1) The optimization model of the system-level

$\operatorname{Min}: f=z_{1}^{2}+z_{2}^{2}$

s.t. $J_{1}^{*}=\left(x_{11}-z_{1}\right)^{2}+\left(x_{12}-z_{2}\right)^{2}=0$

$J_{2}^{*}=\left(x_{21}-z_{1}\right)^{2}+\left(x_{22}-z_{2}\right)^{2}=0$

where, $z_{1}, z_{2}$ are the system-level design variables, $J_{1}^{*}$, $J_{2}^{*}$ are consistency constraints.

2) The optimization model of the disciplines 1

Min : $J_{1}=\left(x_{11}-z_{1}\right)^{2}+\left(x_{12}-z_{2}\right)^{2}$

s.t. $x_{1}+\beta x_{2}<4$

3) The optimization model of the disciplines 2

Min: $J_{2}=\left(x_{21}-z_{1}\right)^{2}+\left(x_{22}-z_{2}\right)^{2}$

s.t. $\beta x_{1}+x_{2}>2$

We can obtain that, when $\beta=0.11$, the optimal solution $x^{*}$ is $(0.191,1.950)$, and the objective function $f^{*}$ is 3.93 . Considering two different starting points, the optimization results of SA-ANN-CO are shown in Table $\mathbf{1}$.

From the Table 1, we can see that SA-ANN-CO can converge to the optimal solutions. The iterations are carried out 30 times to obtain the objective value. The convergence efficiency of SA-ANN-CO is very good. Figs. (4 and 5) show the system optimization goals and the number of iterations of SA-ANN-CO at the initial point 1 and the initial point 2 .

\subsection{Examples 2}

Example 2 is a coupling function optimization problem. The mathematical model is as follows:

Min : $f(x)=x_{1}^{2}+x_{3}+x_{4}+e^{-x_{2}}$

s.t. $g_{i}(x)<0 \quad i=1,2, \cdots 7$
Table 1. The result of optimization results.

\begin{tabular}{|c|c|}
\hline Optimization Methods & SA-ANN-CO \\
\hline \hline The initial point 1 & $(1.1,0.1)$ \\
\hline The initial values & $(0.210,1.8936)$ \\
\hline Optimal feasible solution & 4.013 \\
\hline Optimization target & $5.90 \times 10^{-11}$ \\
\hline Consistency constraints $J_{1}^{*}$ & $5.11 \times 10^{-9}$ \\
\hline Consistency constraints $J_{2}^{*}$ & 30 \\
\hline System-level iterations & $(-1.05,-3.11)$ \\
\hline The initial point 2 & 9.5 \\
\hline The initial values & $(0.202,2.010)$ \\
\hline Optimal feasible solution & 4.015 \\
\hline Optimization target & $5.90 \times 10^{-11}$ \\
\hline Consistency constraints $J_{1}^{*}$ & $5.11 \times 10^{-9}$ \\
\hline Consistency constraints $J_{2}^{*}$ & 30 \\
\hline System-level iterations & \\
\hline
\end{tabular}

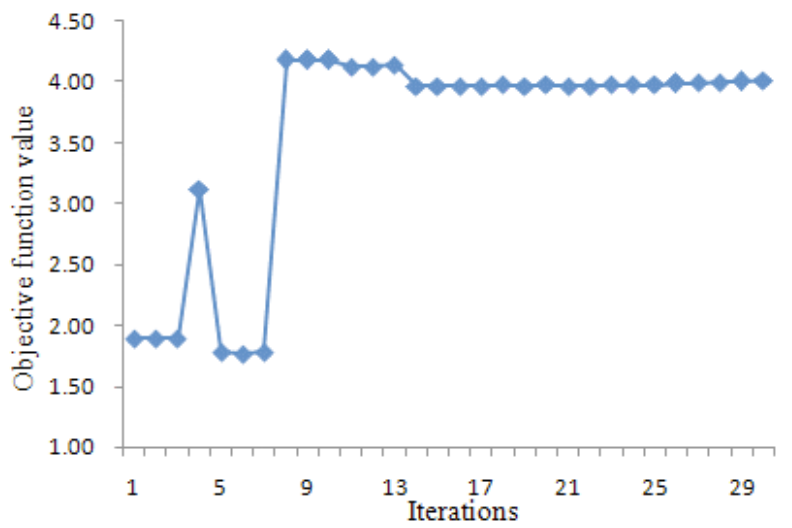

Fig. (4). The system-level target iterative process of SA-ANN-CO at the initial point 1 .

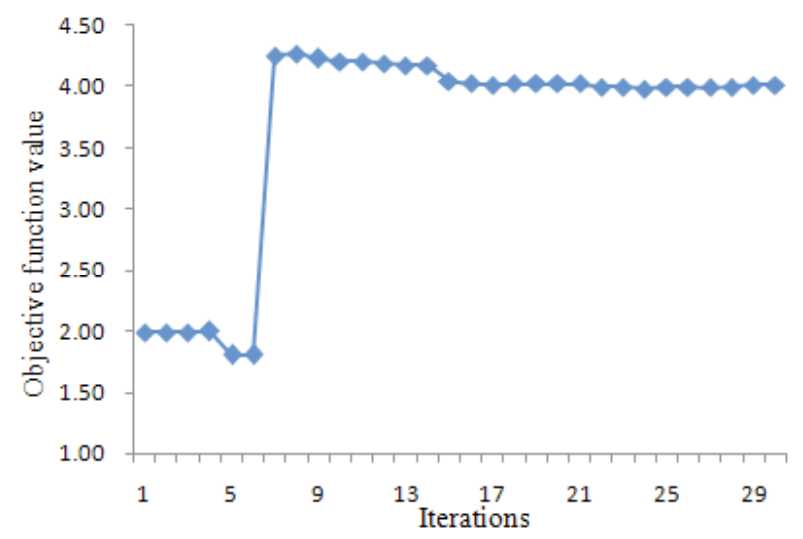

Fig. (5). The system-level target iterative process of SA-ANN-CO at the initial point 2 . 
where:

$$
\begin{aligned}
g_{1} & =x_{4}-x_{1}^{2}-x_{2}-x_{3}+0.2 x_{5}=0 \\
g_{2} & =x_{5}-x_{4}-x_{1}-x_{3}=0 \\
g_{3} & =-x_{4}+1 \leq 0 \\
g_{4} & =x_{5}-1 \leq 0 \\
g_{5} & =x_{1}^{2}-100 \leq 0 \\
0 & \leq g_{6}=x_{2} \leq 10 \\
0 \leq g_{7} & =x_{3} \leq 10
\end{aligned}
$$

According to the idea of Collaborative Optimization, the optimization problem is divided into a system-level and two disciplines. $g_{1}, g_{3}, g_{5}, g_{6}$ are the constraints of discipline 1, $g_{2}, g_{4}, g_{7}$ are the constraints of discipline 2. The optimization model is as follows:

1) The optimization model of the system-level:

$$
\begin{aligned}
& \text { Min: } f=z_{1}^{2}+z_{3}+z_{4}+e^{-z_{5}} \\
& \text { s.t. } J_{1}^{*}=\left(x_{1}-z_{1}\right)^{2}+\left(x_{2}-z_{2}\right)^{2}+\left(x_{3}-z_{3}\right)^{2}+\left(x_{4}-z_{4}\right)^{2}+\left(x_{5}-z_{5}\right)^{2}=0 \\
& J_{2}^{*}=\left(x_{1}-z_{1}\right)^{2}+\left(x_{3}-z_{3}\right)^{2}+\left(x_{4}-z_{4}\right)^{2}+\left(x_{5}-z_{5}\right)^{2}=0
\end{aligned}
$$

2) The optimization model of the disciplines 1

Min : $J_{1}=\left(x_{1}-z_{1}\right)^{2}+\left(x_{2}-z_{2}\right)^{2}+\left(x_{3}-z_{3}\right)^{2}+\left(x_{4}-z_{4}\right)^{2}+\left(x_{5}-z_{5}\right)^{2}$ $g_{i}(x)<0 \quad i=1,3,5,6$

3 ) The optimization model of the disciplines 2

$$
\text { Min : } J_{2}=\left(x_{1}-z_{1}\right)^{2}+\left(x_{3}-z_{3}\right)^{2}+\left(x_{4}-z_{4}\right)^{2}+\left(x_{5}-z_{5}\right)^{2}
$$

$g_{i}(x)<0 \quad i=2,4,7$

Considering two different starting points, the optimization results of SA-ANN-CO shown in Table 2:

Table 2. Comparison of the optimization results.

\begin{tabular}{|c|c|}
\hline Optimization Methods & SA-ANN-CO \\
\hline \hline The initial point 1 & $(0.2,0.2,0.2,0.2,0.2)$ \\
\hline The initial function values & 1.1 \\
\hline Optimal feasible solution & $(0.0713,1.26,-0.091,0.92,1.12)$ \\
\hline Optimization target & 1.210 \\
\hline The initial point 1 & $(3.1,3.1,3.1,3.1,3.1)$ \\
\hline The initial function values & 15.2 \\
\hline Optimal feasible solution & $(0.076,1.31,-0.091,0.092,1.05)$ \\
\hline Optimization target & 1.16937 \\
\hline
\end{tabular}

As can be seen from the Table 2 , the results of SA-ANN$\mathrm{CO}$ is reduced about $14 \%$ at different starting points, with satisfying the consistency constraints, which indicates that SA-ANN-CO is insensitive to the initial point and has global convergence.

\section{CONCLUSION}

We propose a Collaborate Optimization based on Simulated Annealing and Artificial Neural Networks, (SA-ANN$\mathrm{CO})$. The proposed algorithm has the following advantages:

1) Simulated Annealing algorithm is applied in the system level, which avoiding the problems occurred in classical mathematical optimization algorithm;

2) A high degree of consistency for shared variables between system-level and disciplines level variables in line with the actual engineering applications' goal that the design variables of various disciplines must be common;

3) Artificial neural network model is used to replace the real models of disciplines, which will not only meet the reliability requirements, but also reduce the computational cost.

Two classic examples show that, SA-ANN-CO algorithm can quickly and effectively find the global optimal solution. For the initial point in feasible domain, we can combine Simulated Annealing algorithm with gradient-based optimization algorithm to solve issues of high calculation cost. In addition, SA-ANN-CO algorithm also requires testing and further improved in large complex engineering system design.

\section{CONFLICT OF INTEREST}

The authors confirm that this article content has no conflict of interest.

\section{ACKNOWLEDGEMENTS}

Declared none.

\section{REFERENCES}

[1] J. Sobieszczanski-Sobieski, and R.T. Haftka, "Multidisciplinary aerospace design optimization: survey of recent developments," Structural and Multidisciplinary Optimization, vol. 14, no. 1, 1997, pp. 1-23.

[2] I. Kroo, S. Altus, R. Braun, R, P. Gage, and I. Sobieshi, "Multidisciplinary Optimization Methods for Aircraft Preliminary Design", AIAA Paper, 1994, p. 4325.

[3] J. Agte, O. de Weck, J. Sobieszczanski-Sobieski, P. Arendsen, A Morris, and M. Spieck, "MDO: assessment and direction for advancement - an opinion of one international group," Structural and Multidisciplinary Optimization, vol. 40, no. 1-6, pp. 17-33, 2010.

[4] R. D. Braun, A. A. Moore, and I. M. Kroo, "Use of the collaborative optimization architecture for launch vehicle design," In: Proceedings $6^{\text {th }}$ AIAA/USAF/NASA/ISSMO Symposium on Multidisciplinary Analysis and Optimization, 1996, pp. 306-318.

[5] H. Li, M. X. Ma, Z. Huang, and Y. Jing, "New adaptive penalty scheme for collaborative optimization," Journal of System Simulation, vol. 12, no. 19, pp. 6178-6182, 2009.

[6] H. Li, Y.W. Jing, and W. L. Zhang, "A new collaborative optimization based on genetic algorithm," Journal of Northeastern University, vol. 32, no. 8, pp. 1074-1077, 2009.

[7] H. Ling, Y. Cheng, J. Liu, and G. Zen, “An improved multidisciplinary collaborative optimization algorithm and its application in 
submarine subdivision," Shipbuilding of China, vol. 52, no. 2, pp. 87-99, 2011.

[8] Z. Xia, Y. Liu, W. Fan, and W. Wang, "Method study of collaborative optimization with mixed variables," Journal of System Simulation, vol. 23, no. B07, pp. 55-58, 2011.

[9] F. Scarselli, and T. A. Chung, "Universal approximation using feedforward neural networks: A survey of some existing methods, and some new results," Neural Networks, vol. 11, no. 1, pp. 15-37, 1998.
[10] A. Corana, M. Marchesi, C. Martini, and S. Ridella, "Minimizing multimodal functions of continuous variables with the "simulated annealing" algorithm Corrigenda for this article is available here," ACM Transactions on Mathematical Software (TOMS), vol. 13, no. 3, pp. 262-280, 1987.

[11] B. Roth, and I. Kroo, "Enhanced collaborative optimization," In: $12^{\text {th }}$ AIAA/ISSMO Multidisciplinary Analysis and Optimization Conference, Victoria, BC, 2008.

Received: June 10,2015

(C) Qiang and Zhao; Licensee Bentham Open

This is an open access article licensed under the terms of the Creative Commons Attribution Non-Commercial License (http://creativecommons.org/licenses/by-nc/3.0/) which permits unrestricted, non-commercial use, distribution and reproduction in any medium, provided the work is properly cited. 